\title{
Diagnóstico del dominio del inglés en el mercado laboral turístico de Puerto López
}

\section{Diagnostic of English proficiency in the tourism labor market of Puerto Lopez}

José Oswaldo Tigua Anzules Mg. EII ${ }^{(1)}$

Marianela San Lucas Marcillo Mg.EII ${ }^{(2)}$

Marieta Azúa Menéndez. Mg.EI ${ }^{(3)}$

$\left({ }^{1,2,3}\right)$ Centro de Idiomas. Universidad Estatal del Sur de Manabí. Ecuador

Contacto: josetg28@hotmail.es

Receptado: 24/04/2017

\section{Resumen}

La presente investigación se realizó en la cabecera cantonal de Puerto López de la Provincia de Manabí, básicamente a los empresarios y Guías de Agencias de viajes. El diagnóstico del dominio del idioma Inglés tiene como propósito averiguar el nivel de conocimiento que adquieren los autores turísticos en el mercado laboral del mismo. Indicando que es de conocimiento que una buena comunicación es la mejor herramienta para impulsar las estrategias de internacionalización, es por eso que el aprendizaje del idioma inglés, cada día toma más fuerza, actualmente ya no es un lujo si no una obligación dominar una segunda lengua permitiendo que muchas puertas se abran en el sector laboral y en especial en el turístico, de acuerdo a los métodos utilizados en esta investigación fueron: el histórico-lógico, inductivo-deductivo y la observación, encuestas a empresarios turísticos y guías de agencias de viajes. Las pequeñas, medianas y grandes empresas actualmente cuentan con pocas personas que dominen este idioma haciendo que se pierdan oportunidades en el campo laboral. La globalización esta generado cambios en la economía, los negocios y el campo laboral de muchos países entre ellos el nuestro. A medida que el entorno se hace más global, es necesario el dominio de una segunda lengua para afrontar con éxito los retos que demanda la actividad económica y profesional de este siglo.

Palabras claves: Diagnóstico, oportunidades laborales, aprendizaje, dominio 


\section{Abstract \\ Diagnostic of English proficiency in the tourism labor market of Puerto Lopez}

This research was conducted in the cantonal center of Puerto Lopez Manabí Province, mainly entrepreneurs and guides Travel agencies. Diagnosis of English language proficiency is to ascertain the level of knowledge among tourist authors in the labor market the same purpose. Indicating that is aware that good communication is the best tool for promoting internationalization strategies is why the English language, each day takes more strength, is now no longer a luxury but a requirement to master a second language since this allows many doors open to in the labor sector, especially in tourism, according to the methods used in this research were: the historical and logical, inductive-deductive and observation, surveys tourism operators and guides travel agency. Small, medium and large enterprises currently have few people who master this language by missed opportunities in the workplace. Globalization is generated changes in the economy, business and the workplace in many countries including ours. As the environment becomes more global, the domain of a second language is required to successfully meet the challenges entailed in economic and professional activity of this century.

Keywords: diagnostic, Jobs opportunities, learning, command.

\section{Introducción}

El idioma inglés es de gran importancia a nivel mundial ya que aporta a la preparación integral del hombre actual. Durante los últimos años las necesidades comunicativas especializadas y dirigidas a las distintas profesiones están basadas en los nuevos perfiles de las diferentes empresas, ya que la demanda laboral crece día a día.

Sin embargo, muchas entidades reconocen que encuentran inconvenientes a la hora de comunicarse con sus clientes potenciales en el Idioma Inglés, ya que se ha convertido en la lengua franca para los negocios internacionales. Como es de conocimiento, una buena comunicación es el mejor instrumento para impulsar las estrategias de internacionalización. Por tal razón Jahir Lombana considera que "El inglés es 
la clave en el mundo de los negocios puesto que en la mayoría de los casos el idioma con el que se realiza el trato es la efectividad con que se concreta".

Según (Riesco, 2012) señala que la necesidad de abordar una enseñanza en inglés eficaz, tanto desde las universidades y escuelas de negocios como desde la propia empresa. Esta formación debe dotar a los estudiantes de las herramientas del idioma necesarias para abordar situaciones reales de negociación, presentación de empresas y productos. Además, se incluye también a las compañías europeas que utilizan el inglés como 'idioma de todos’, sin estar ligado a ninguna nacionalidad en concreto.

Durante los últimos años, la globalización ha tenido enormes cambios en la economía, los negocios y el campo laboral especialmente en el campo turístico. A medida que el entorno va creciendo este se hace más global por tal razón es necesario aprender y dominar una segunda lengua para afrontar con éxito los retos que demanda la actividad económica y profesional de este siglo. Cabe resaltar que el idioma que ha adquirido máxima importancia en la comunicación alrededor del mundo es el inglés.

Con relación a los negocios y la economía, desde principios del siglo XX el inglés fue tomando un papel importante y se convirtió en el idioma universal. En la actualidad muchas empresas exigen hoy hablar inglés para acceder a puestos laborales. La mayoría de estudios y textos científicos están escritos en este idioma. Y si se habla de tecnología e informática, gran parte de la terminología o vocabulario especializado es en inglés. En el mundo de Internet, muchas veces la información redactada en inglés supera a la información disponible en español. Más de 400 millones de personas tienen al inglés como lengua materna; el número se incrementa si se toma como referencia a aquellos países, en donde el inglés es considerado como segunda lengua.

Expertos del ICEX (Instituto de Comercio Exterior), de las Cámaras de Comercio de Madrid y Barcelona, coincidieron en la importancia de la internacionalización de las sociedades de cualquier tamaño para hacer frente a la actual crisis económica. Sin embargo, la capacidad para comunicarse de forma efectiva en inglés puede marcar la diferencia entre ganar posibilidades de negocio o perderlas.

De igual forma, para (Cordoba, 2011) tomando como aporte de Navarro y Peñeiro (2009, pp.130-131) indican que El idioma inglés ha desarrollado un carácter de lengua franca debido al uso alcanzado en el 
mundo internacional de los negocios. En un ámbito de relaciones comerciales, no solo es considerado el idioma más utilizado, sino también el más hablado.

Álvarez y Bassi (2010, II 2) también consideran que Dominar el inglés como segunda lengua será una habilidad indispensable para acceder a mejores oportunidades en este nuevo siglo. La lengua inglesa es la más útil para la comunicación internacional, interregional y multilateral y es la lengua franca en ámbitos como el mercadeo, el comercio internacional y el turismo.

En la actualidad para las Empresas es fundamental contar en sus filas con personas que puedan mantener una conversación con representantes extranjeros, realizar presentaciones e incluso viajar a países de habla inglesa para cerrar alianzas laborales, sin que el idioma sea un impedimento. Además, tener grupos de trabajo bilingües significa un ahorro monetario, ya que no se necesita contratar traductores o intérpretes para poder realizar o incluso entender los planes de negocio a emprender.

El nivel de inglés de América Latina es muy bajo. En parte se explica por la importancia y predominio del español en la región, siendo una lengua compartida que permite el comercio continental, la diplomacia y los viajes, reduciendo la motivación para aprender inglés. Según el estudio Education first English proficiency index (EF EPI), Chile se posiciona en el lugar $39^{\circ}$ de un ranking mundial de dominio de inglés, aplicado en países de habla no anglosajona. En la región se encuentra por debajo de Argentina, Uruguay, Perú, Costa Rica y México.

Por todas estos inconveniente sobre el aprendizaje del Inglés se sigue insistiendo en la necesidad creciente en la sociedad moderna de un buen conocimiento de lenguas extranjeras para las relaciones internacionales por diversos motivos (educativos, laborales, profesionales, culturales o turísticos), para tener acceso a medios de Comunicación y para el desarrollo de las nuevas tecnologías. .

En Ecuador el aprendizaje integrado de lenguas y contenidos a través de una segunda lengua requiere la participación activa de los actores del habla inglesa, más aún en los lugares turísticos en donde la afluencia de extranjeros ha aumentado, por tal razón es necesario el aprendizaje de este idioma y así poder comunicarse con mayor facilidad. 
De acuerdo a (Martinetti \& Choez, 2015) carecer de un conocimiento básico lingüístico de los países asiáticos o del idioma como lenguaje intermedio, se genera un factor negativo en un entorno internacional, sobre todo el mercado cacaotero, ya que en este la competencia es alta tanto a escala nacional como internacional. Carecer de un departamento de Traducción e Interpretación, (T\&I) que cuente con las competencias interculturales necesarias, puede representar una desventaja para que las empresas ecuatorianas ingresen a este mercado.

Estos autores indican que por el poco aprendizaje en este idioma se pueden obstaculizar muchos negocios importantes, por el simple hecho de no saber o no tener conocimiento del inglés en el ámbito empresarial.Según (Ramos \& Ruiz, 2011). El Libro Guinness de los Records, el inglés es la segunda lengua más hablada del mundo (el español es la cuarta), por detrás del chino mandarín, que cuenta con más de un billón de hablantes. Además para el uso de las plataformas digitales, también es primordial el inglés, dando a entender que es necesario y fundamental aprender el inglés en la sociedad actual.

Según (Buitrago Sanabria, 2011) Indica que la importancia de adquirir una lengua extranjera, hace que se convierta en una competencia comunicativa, utilizando algunos métodos para la enseñanza de una lengua extranjera y a la vez acceder a una formación virtual.

A la vez Según (Niño, 2013) considera que para sobrevivir en este mundo competitivo, todos debemos ser capaces de asumir nuevos retos y oportunidades indicando que si no se esfuerzan en aprender se quedaran rezagados de las oportunidades que se les presenten. En el caso de las empresas turísticas como hoteles, restaurantes ponen carteles sean estos de bienvenidas, despedidas, agradecimientos o en el caso el menú en ingles indicando que tienen poco conocimiento del inglés y a veces esto hace que pierdan clientes.

Entendiendo que por motivo de esta creciente necesidad de aprender este idioma, las empresas han optado también por implementar planes de capacitación empresarial en un segundo idioma. Según la medición incluida en el informe "Formación online de las competencias comunicativas para una fuerza laboral internacional muy diversa", desarrollado por la firma digital publishing - dp y presentado durante los primeros días de marzo de 2012, ocho de cada diez organizaciones (79\%) considera que el aprendizaje de idiomas va a ser un foco principal en sus planes de desarrollo laboral. 


\section{Rev. SINAPSIS, Edición № 10, Vol. 1, Junio 2017}

Como se pudo ver a lo largo del artículo, hoy en día es necesario la adquisición del idioma como segunda lengua ya que nos ayuda a encontrar un mejor empleo y aún más en el ámbito turístico, considerando que Ecuador en especial Puerto López se están convirtiendo en un potencial turística atrayendo muchos extranjeros y también mucha fuente de trabajo.

Por tal razón lo expuesto por (Riesco, 2012) indica que esta formación debe dotar a los estudiantes de las herramientas del idioma necesarias para abordar situaciones reales de negociación, presentación de empresas y productos. Ya que todos los equipos de trabajo de las empresas en especial las dedicadas al comercio internacional deben contar con personal altamente calificado y con conocimientos netamente académicos en manejar otras lenguas, en especial el inglés que un requisito indispensable para poder desarrollar las funciones laborales efectivamente.

La causa de estas manifestaciones es la escasa motivación por parte de los empleados en aprender este idioma y no lo reconocen como una lengua importante. El objeto se basa Diagnóstico del dominio del inglés en el mercado laboral turístico de Puerto López. Como Objetivo de esta investigación es diagnosticar el nivel de conocimientos del idioma inglés para las personas que ingresen a trabajar una empresa Turística mediante una encuesta.

En este proceso influye mucho la enseñanza y aprendizaje que recibieron en sus años de estudios lo importante no es tanto la lengua extranjera sino el desarrollo de las capacidades comunicativas de la misma. El campo, es el aprendizaje del inglés con enfoque empresarial en el área turística y para así contribuir al desarrollo socio-económico del Cantón, ya que muchas personas se dedican a diferentes actividades laborales. Para fundamentar la relación y el uso de estos contenidos los actores deben prepararse para así poder ser más competitivos.

\section{Materiales y métodos}

Para observar el comportamiento de la comunicación y las necesidades comunicativas de los emprendedores turísticos se realizaron encuestas y se entrevistaron a varios empresarios de la localidad y se aplicó un instrumento de diagnóstico para determinar el dominio del idioma ingles y con ello se conocieron las insuficiencias en las competencias lingüísticas del mercado laboral turístico de la ciudad de Puerto López. 
La población estuvo constituida por 5 empresas hoteleras, 3 agencias turísticas en total del cual fueron encuestada 20 personas relacionadas al turismos. Estas encuestas nos permitieron conocer el nivel de conocimientos de las habilidades comunicativas por los emprendedores y también la muestra (20) se determinó que algunos de los empresarios, empleados y emprendedores tienen conocimiento básico del inglés, recalcando que un pequeño grupo maneja muy bien este idiomas y ellos han triunfados en sus negocios, gracias a su preparación y vastos conocimientos

Este diagnóstico se partió de una sistematización teórica en las temáticas más relevantes en lo laboral, tomándose en consideración los antecedentes relacionados con el idioma inglés en el sector laboral turístico.

Los métodos que se utilizaron fueron: El método sistémico estructural - funcional se utilizó en la definición del objeto de esta investigación y en la modelación de su concepción teórica, mediante la determinación de los subsistemas que la conforman y las relaciones de cada uno de ellos, así como el establecimiento, estructuración y funcionalidad de la metodología.

El histórico-lógico me ayudo a la determinación de los modelos explicativo-descriptivos usados en la estrategia de la construcción textual escrita del idioma inglés, en la disciplina del objeto de estudio y de las tendencias actuales de su desarrollo.

Análisis-síntesis e inducción-deducción nos permitieron interpretar la información bibliográfica y de los datos estadísticos; además, posibilitaron la caracterización del objeto y el campo a partir del análisis de los fundamentos teóricos que los sustentan.

De los métodos empíricos: se aplicó la observación en el área de trabajo, encuestas, para evaluar el estado actual de las destrezas comunicativas del inglés y su aplicación en área de turismo.

\section{Resultados}

Como resultado de las encuestas realizadas para esta investigación nos indicaron las personas encuestadas que si están interesadas en mejorar y dominar este idioma ya que les permite un mejor desenvolvimiento en sus actividades laborales, además les permite manipular con facilidad este idioma. Los empresarios y guías de agencias de viajes relacionan el aprendizaje del idioma ingles como una herramienta importante en el ámbito laboral. 
El propósito es obtener información para verificar las condiciones en que ellos han desarrollado las habilidades comunicativas en el idioma inglés antes de ingresar al mundo laboral.

En la tabla No.1 hace referencia de que si ¿considera usted que el dominio del idioma ingles es fundamental para acceder a una propuesta laboral?

Tabla No. 1

\begin{tabular}{lccc}
\hline & Alternativa & Frecuencia & Porcentaje \\
\hline $\mathrm{Si}$ & 15 & $75 \%$ \\
$\mathrm{No}$ & 4 & $20 \%$ \\
No Sabe/No Contesta & 1 & $5 \%$ \\
\multicolumn{2}{r}{ Total } & 20 & $100 \%$ \\
\hline
\end{tabular}

Fuente: "Empresarios y Guías de Agencias Turísticas del Cantón Puerto López".

Durante el período de investigación y aplicando encuestas a los empresarios y guías Agencias de Viajes en la Ciudad de Puerto López, fueron corroborados a partir de la encuesta realizada se pudo constatar que de 20 Empresarios (población), 20 (muestra) fueron encuestado en el Cantón Puerto López, donde se elaboraron figuras con datos relacionados con las variables que a continuación se detallan: En la figura No.1 hace referencia de que si ¿considera usted que el dominio del idioma ingles es fundamental para acceder a una propuesta laboral? y, de acuerdo a su tabulación se determina que un $75 \%$ manifestó que si, un $20 \%$ dijo que no y un $5 \%$ no sabe no contesta, por lo que indica que ellos si presentan interés en el dominio de este idioma extranjero (Inglés) ya que piensan es necesaria para sus actividades laborales.

Continuando con el análisis de la tabla No. 2 referente a ¿Posee ud conocimientos necesarios en el idioma ingles para poder interactuar con una persona de habla inglesa?

Tabla No. 2

\begin{tabular}{lccc}
\hline & Alternativa & Frecuencia & Porcentaje \\
\hline $\mathrm{Si}$ & 3 & $15 \%$ \\
\hline
\end{tabular}


Rev. SINAPSIS, Edición № 10, Vol. 1, Junio 2017

\begin{tabular}{rcc}
\hline No & 16 & $80 \%$ \\
No Sabe/No Contesta & 1 & $5 \%$ \\
Total & 20 & $100 \%$ \\
\hline
\end{tabular}

Fuente: "Empresarios y Guías de Agencias Turísticas del Cantón Puerto López".

Continuando con el análisis de la tabla No. 2 referente a si ¿Posee Ud. conocimientos necesarios en el idioma ingles para poder interactuar con una persona de habla inglesa? muestra como resultado que un $15 \%$ manifiestan que si posee el conocimiento necesario para empezar una conversación con un extranjero del habla inglesas, un $80 \%$ dice que no tiene conocimiento en este idioma (Ingles) y un 5\% No sabe/No contesta. Cabe indicar que las habilidades comunicativas se consideran fundamental a la hora de aprender una nueva lengua.

En la tabla No. 3 se analiza los resultados de la pregunta ¿Relaciona el aprendizaje del idioma ingles como una herramienta importante en el ámbito laboral?

\section{Tabla No. 3}

\begin{tabular}{lccc}
\hline & Alternativa & Frecuencia & Porcentaje \\
\hline $\mathrm{Si}$ & 18 & $90 \%$ \\
$\mathrm{No}$ & 1 & $5 \%$ \\
No Sabe/No Contesta & 1 & $5 \%$ \\
\multicolumn{1}{r}{ Total } & 20 & $100 \%$ \\
\hline
\end{tabular}

Fuente: "Empresarios y Guías de Agencias Turísticas del Cantón Puerto López".

En la encuesta realizada a los Empresarios y Guías de Agencias de viaje se pudo conocer que el $90 \%$ relaciona el aprendizaje del idioma ingles como una herramienta importante en el ámbito laboral, un 5\% consideraron que no, ya que hay extranjeros que también saben el español o cargan traductores con el cual pueden consultar lo que quieren saber o preguntar, y un 5\% no sabe, no contesta o no desean aprender.

En cuanto al dominio de las habilidades del idioma inglés se pudo comprobar en las encuestas realizadas que la población antes mencionada posee un grado de conocimiento básico en esta lengua. Los propietarios y servidores de empresas Turísticas expresaron que para comunicarse con los turistas deben 
aprenderlo ya que los turistas manejan un inglés fluido por lo tanto los empresarios y empleados deben mejorar sus conocimientos básicos en este idioma.

De los encuestados un tanto por ciento no poseen conocimientos de esta lengua pero la interrelación con los turistas de habla inglesa le ha permitido comprender y entender sus necesidades. Al aplicar el instrumento para conocer el segundo indicador de este diagnóstico basado en la motivación de los trabajadores para la superación del idioma inglés, consideran que se sienten apto y predispuestos a recibir capacitaciones por la alta demanda que tiene el Cantón, ya que está relacionada con visitas de turistas extranjeros y esto hace que el aprendizaje sea más fácil para ellos, porque se dan cuenta las falencias que tienen como personas encargadas de atender al turista ya que este es su trabajo, y como recompensa tendrán un turismo permanente y sus ingresos económicos mejorarían y la comunicación será positiva.

De acuerdo ITUR Puerto López (servicio de información y atención al turista) indica que en el transcurso de estos últimos 3 años la visita de extranjeros especialmente del habla inglesa ha sido favorable más aun para la temporada de Ballena que inicia desde junio hasta septiembre, aportando así él porque es necesario aprender este idioma.

\section{Tabla No. 4}

Total De Turista Por Años.

\begin{tabular}{|l|r|}
\multicolumn{1}{|c|}{ Año 2013 } & $\mathbf{6 5 5 8}$ \\
\hline Año 2014 & 18144 \\
\hline Año 2015 & 15428 \\
\hline
\end{tabular}

Fuente: "Empresarios y Guías de Agencias Turísticas del Cantón Puerto López".

\section{Discusión}

En esta investigación se pudo comprobar que todas las personas inmersas en el sector laboral necesitan aprender inglés como una herramienta muy importante en su desempeño laboral, También podemos decir que las nuevas tecnologías han provocado un cambio sustancial en el perfil del profesional y a su vez han añadido valor a los servicios que ofrecen al usuario. 
El auge de este nuevo segmento del sector turístico cultural tiene repercusiones económicas positivas, no sólo en el empleo, sino también en todos aquellos aspectos relacionados con el turismo en general transporte, hostelería, restauración indica según (Piedrola \& Artacho, 2011). De acuerdo a la expectativa del autor es real ya que si uno no cumple bien en su trabajo y no se esfuerza en aprender el idioma inglés esto hará que su labor no sea gratificante pero si el analiza los cambios que se pueden dar, por saber este segundo idioma se beneficiarán el sector turístico como los visitantes extranjeros en especial los de habla inglesa.

De acuerdo a (Avalos \& Zapata, 2014) observa una alta exigencia de competencias genéricas; es decir, destrezas, habilidades, conocimientos y actitudes básicas o fundamentales para el desempeño laboral como: habilidad de trabajar en equipo, habilidad para comunicarse efectivamente de manera oral y escrita.

Según (Bonilla L. A., 2012) El aprendizaje de idiomas extranjeros, si bien es conocido, trae consigo ventajas competitivas para quien lo domine ante los demás ya que es una actividad desafiante y atractiva a cualquier edad, su conocimiento facilita obtener y abrir nuevas oportunidades de desarrollo el cual se convierte en una valiosa herramienta de trabajo y en un medio de acceso al conocimiento universal y la cooperación entre los pueblos.

A medida que se producen estas modificaciones, se debe mantener un balance entre cambio y estabilidad para asegurar el mejor diseño de los servicios. Todas las tareas y responsabilidades que desempeña una persona en muchos casos son modificadas por la tecnología.

(Roa, Noviembre 2013) indica que según Para Alfonzo, K. (2006) en su trabajo de investigación titulado: "Importancia de la comprensión y producción oral del inglés como lengua extranjera en el reclutamiento de personal" afirma que: "el inglés como segunda lengua es una herramienta esencial para el hotelero, ya que la producción oral y comprensión auditiva son de vital importancia para el buen desempeño en el campo laboral para tener la oportunidad de un trabajo en el ámbito del turismo en el extranjero es obligatorio el manejo de un nivel intermedio del idioma inglés." (p 52).

(Martinez, 2013) En el trabajo de investigación, coge como referencia a Peña, D. (2011) donde afirma que: "La comunicación desempeña un papel importante en el éxito de una estrategia de servicio, es el 


\section{Rev. SINAPSIS, Edición № 10, Vol. 1, Junio 2017}

vehículo indispensable para ampliar la clientela, conseguir lealtad, motivar a los empleados y darles a conocer las normas de calidad que deben poner en práctica.” (p. 47),

De acuerdo a los Autores Alfonzo y Martínez coinciden que para una buena oportunidad de trabajo, es necesario tener conocimiento del manejo del idioma inglés, resaltando así que la comunicación interviene en el logro de las tácticas de servicio, siendo ésta fundamental para generar una estabilidad organizacional.

El mercado laboral actual se enriquece cada día con las nuevas tecnologías, para ello los profesionales deben ser suficientemente abiertos y flexibles a los cambios. Además, el este mercado exige una modificación en el perfil del profesional acorde a la época y es por eso que la enseñanza - aprendizaje de este idioma busca un desarrollo integral de todas las habilidades que permitan el intercambio académico, cultural y profesional con personas de otros países y así poder desarrollar de una mejor manera su trabajo.

\section{Conclusiones}

Los resultados de esta investigación permitieron determinar la necesidad de aplicar un sistema de acciones de capacitación dirigida a la interpretación de las 4 habilidades (listening, speaking, reading and writting) y al léxico con fines turísticos que contribuyan a establecer una comunicación afectiva con el visitante extranjero.

1. Los trabajadores de la comercialización del producto turístico y las relaciones públicas no poseen en su gran mayoría formación académica en el idioma y aún tienen limitaciones en las habilidades de la lengua y el léxico especializado lo que incide negativamente en su nivel profesional integral y en la efectividad de la comunicación.

2. Se demostró la necesidad de aplicar un sistema de acciones de capacitación dirigidas a la integración de las cuatro habilidades básicas en Lengua Inglesa y al léxico con fines profesionales a los trabajadores del área comercial y las RRPP que contribuyan a establecer una comunicación efectiva con el cliente extranjero.

3. Se demostró la necesidad de diseñar una Campaña de RRPP para comunicar las acciones a los públicos internos y externos a través de diferentes canales.

4. Liderar la enseñanza del inglés desde los diferentes entornos, mediante las nuevas tecnologías, sin perder la identidad nacional y el compromiso con la lengua materna. Se trata de garantizar a 
la comunidad mejores posibilidades de insertarse en el mundo globalizado, conocer otras culturas y desempeñarse con el apoyo de otras lenguas y desde esta vía apoyar la equidad y la igualdad de oportunidades.

\section{Bibliografía}

Avalos, A. c., \& Zapata, N. M. (2014). Competencia requeridas en el ámbito laboral del turismo. Revista electónica F pune Scientific, 157-158.

Bonilla, L. A. (2012). El aprendizaje de lenguas extranjeras como herramientas para el desarrollo humano. Las lenguas en la educacion, 2-11.

Buitrago Sanabria, A. (2011). Ambiente virtual de aprendizaje: Una herramienta para mejorar la comprension y uso del ingles. Uniminuto, 8-134.

Cordoba, G. G. (2011). Enseñanza y Aprendizaje del ingles y las pobrezas, 9-30.

Martinetti, N., \& Choez, M. (2015). Importancia de la traduccion e interpretacion de idiomas para las empresas exportadoras de cacao. Retos, 3-11.

Martinez, M. (2013). Importancia de las destrezas linguisticas en el idioma ingles para el aseguramiento de la calidad de la prestacion del servicio en el Departamento de comercializaión y ventas. Colegio Universitario Hotel Escuela de los Andes Venezolano, 50- 98.

Niño, P. M. (2013). El Inglés y su importancia en la Investigacion Científica. Colombiana ciencia Anim., $7-8$.

Piedrola, O. I., \& Artacho, R. C. (2011). El turimo Idiomático= una oportunidad de desarrollo local para Cordoba. Análisis turístico, 53-61.

Ramos, F., \& Ruiz, O. J. (2011). La educacion fisica en centros bilingues de primaria Ingles - Español: De las singularidades propias del area a la Elaboracion de propuestas didacticas practicas con AIBLE. RESLA 24, 1 - 18.

Riesco, S. (2012). Ingles, Idioma de las empresas y negocios. Funiber, 1-5. 
Rev. SINAPSIS, Edición №10, Vol. 1, Junio 2017

Roa, I. (Noviembre 2013). Necesidades del idioma Inglés como aprendizaje para los empleados que laboran en el area de recepción del hotel kactus, ubicado en Barina Edo. Colegio Universitario Hotel escuela de los Andes Venezolanos sudirección Académica., 20-58.

Niño, P. M. (2013). El Inglés y su importancia en la Investigacion Científica. Colombiana ciencia Anim., $7-8$.

ITUR. Puerto López (servicio de información y atención al turista) 2016 